\title{
Prospects for Open Heavy Flavor Measurements in Heavy Ion and $p+A$ Collisions in a Fixed-Target Experiment at the LHC
}

\author{
Daniel Kikoła \\ Faculty of Physics, Warsaw University of Technology, Ulica Koszykowa 75, 00-662 Warsaw, Poland \\ Correspondence should be addressed to Daniel Kikoła; kikola@if.pw.edu.pl
}

Received 20 March 2015; Accepted 24 May 2015

Academic Editor: Cédric Lorcé

Copyright (C) 2015 Daniel Kikoła. This is an open access article distributed under the Creative Commons Attribution License, which permits unrestricted use, distribution, and reproduction in any medium, provided the original work is properly cited. The publication of this article was funded by SCOAP .

\begin{abstract}
High luminosity data in a fixed-target experiment allow studying interactions of heavy quarks with nuclear matter in the intermediate energy range with extremely high precision. We present a feasibility study for open charm and bottom production measurements in the energy range of a fixed-target experiment at the LHC (AFTER@LHC). We demonstrate, that high-precision data from AFTER will allow answering two open questions: if there is a collective behavior of charm quarks in $p+A$ collisions at RHIC energy and if charm production is suppressed in the energy range of $\sqrt{s_{N N}}=60-80 \mathrm{GeV}$. We argue that simultaneous measurement of $D^{0}$ suppression as a function of traverse momentum at midrapidity and forward rapidity can help to pin down the mechanism of charm energy loss in the hot and dense nuclear medium.
\end{abstract}

\section{Introduction}

Relativistic heavy ion collisions provide a unique opportunity for studying quark-gluon plasma (QGP), a new state of nuclear matter with properties determined by quark and gluon degrees of freedom. Its properties can be studied with heavy quarks: charm and bottom. They are produced very early in the collision, in the initial interactions with large momentum transfer. Their production and both total and differential cross sections are well described by perturbative QCD calculations. Moreover, due to large masses, they are expected to interact with the nuclear matter differently compared to light partons.

We can infer properties of the QGP by studying modification of heavy quarks production in the heavy ion collisions compared to proton-proton baseline (for details, see [1] and references therein). Energy loss and elliptic flow of open heavy flavor are sensitive to the dynamics of the medium; such measurements could be used to determine the fundamental properties of the QGP, for instance, transport coefficients. Elliptic flow of heavy quarks can give insights into degree of thermalization of the created nuclear matter and can help to discriminate between different models of heavy quark interactions with the QGP.
Heavy flavor production at RHIC (measured via $D^{0}$ and electrons from semileptonic decays of heavy flavor hadrons, $\left.e^{\mathrm{HF}}\right)$ in $\mathrm{Au}+\mathrm{Au}$ collisions at $\sqrt{s_{N N}}=200 \mathrm{GeV}$ is suppressed at high transverse momentum $\left(p_{T}\right)$ and $e^{\mathrm{HF}}$ have a significant elliptic flow $v_{2}$ (elliptic flow is defined as the second harmonic in the Fourier expansion of the particle azimuthal distribution with respect to the reaction plane [2]). These results suggest that charm quarks are strongly coupled with the medium: heavy quarks loose a large amount of energy and acquire a significant elliptic flow during interaction with matter created at top RHIC energy.

Beam energy scan (BES) program at RHIC was carried out recently to study the phase diagram of nuclear matter and search for a phase transition and a critical point. BES results show that elliptic flow of inclusive charged hadrons is approximately independent of beam energy (the difference is less than $10 \%$ for $0.5<p_{T}<3 \mathrm{GeV} / \mathrm{c}$ ) and light hadron production is suppressed at high $p_{T}$ in the energy range of $39-200 \mathrm{GeV}$. In $\mathrm{Au}+\mathrm{Au}$ collisions at $\sqrt{s_{N N}}=$ $62.4 \mathrm{GeV}$, production of $e^{\mathrm{HF}}$ is not suppressed (within large uncertainties). Moreover, measurements of elliptic flow of $e^{\mathrm{HF}}$ in $\mathrm{Au}+\mathrm{Au}$ collisions at $\sqrt{s_{N N}}=62.4$ and $39 \mathrm{GeV}$ [3] showed that $v_{2}$ of $e^{\mathrm{HF}}$ is consistent with zero. This is a different 
TABLE 1: Average number of participants $\left\langle N_{\text {part }}\right\rangle$ and binary collisions $\left\langle N_{\text {bin }}\right\rangle$ in $p$-Pb collisions at $\sqrt{s_{N N}}=115 \mathrm{GeV}$ for centrality classes defined as percentiles of the hadronic cross section. The mean values and the RMS are obtained with a Glauber Monte Carlo calculation.

\begin{tabular}{|c|c|c|c|c|c|c|}
\hline Centrality & $b_{\min }[\mathrm{fm}]$ & $b_{\max }[\mathrm{fm}]$ & $\left\langle N_{\text {bin }}\right\rangle$ & $\operatorname{RMS}\left(N_{\text {bin }}\right)$ & $\left\langle N_{\text {part }}\right\rangle$ & $\operatorname{RMS}\left(N_{\text {part }}\right)$ \\
\hline $0-10 \%$ & 0 & 2.41 & 8.0 & 2.7 & 9.0 & 2.7 \\
\hline $10-20 \%$ & 2.41 & 3.42 & 7.3 & 2.6 & 8.3 & 2.6 \\
\hline $20-30 \%$ & 3.42 & 4.19 & 6.6 & 2.4 & 7.6 & 2.4 \\
\hline $30-40 \%$ & 4.19 & 4.83 & 5.8 & 2.3 & 6.8 & 2.3 \\
\hline $40-50 \%$ & 4.83 & 5.41 & 4.9 & 2.1 & 5.9 & 2.1 \\
\hline $50-60 \%$ & 5.41 & 5.93 & 3.9 & 1.8 & 4.9 & 1.8 \\
\hline $60-70 \%$ & 5.93 & 6.43 & 2.9 & 1.5 & 3.9 & 1.5 \\
\hline $70-80 \%$ & 6.43 & 6.95 & 2.2 & 1.2 & 3.2 & 1.2 \\
\hline $80-90 \%$ & 6.95 & 7.60 & 1.6 & 0.8 & 2.6 & 0.8 \\
\hline $90-100 \%$ & 7.60 & 19.80 & 1.2 & 0.4 & 2.2 & 0.4 \\
\hline
\end{tabular}

behavior than that for light hadrons, where a positive $v_{2}$ is observed and the difference between $\sqrt{s_{N N}}=200 \mathrm{GeV}$ and $39 \mathrm{GeV}$ is small. These results suggest that there is a difference in the interactions of the heavy quarks with the surrounding nuclear matter at $200 \mathrm{GeV}$ compared to the two lower energies and there is already a change of the nuclear matter properties in the energy range of 62.4-200 GeV. High luminosity data of a proposed fixed-target experiment at the $\mathrm{LHC}\left(\sqrt{s_{N N}}=72 \mathrm{GeV}\right)$ together with a wide rapidity coverage could shed new light on the energy loss mechanism for charm quarks (which we discuss in Section 5) and give a precise answer if heavy flavor production is suppressed or not at high $p_{T}$.

For the interpretation of the results from heavy ion collisions, it is important to have a good handle on the so-called cold nuclear matter (CNM) effects. This category includes modifications of the particle yields not related to the QGP formation, such as a shadowing (modification of parton distributions in a nucleus) or a Cronin effect (an enhancement of transverse momentum in $p+A$ with respect to $p+p$ collisions). Experiments at RHIC and LHC recently reported collective behavior of light hadrons in high multiplicity $d+$ $\mathrm{Au}$ [4] and $p+\mathrm{Pb}$ collisions [5], where these hadrons have significant elliptic flow with a characteristic mass-splitting pattern [6]. These observations triggered speculations that an enhancement of $e^{\mathrm{HF}}$ production in central and minimum bias $d+$ Au collisions at midrapidity at RHIC [7] could indicate a collective phenomenon (radial flow) of heavy quarks in $d+$ $\mathrm{Au}$ [8]. However, this enhancement could be also due to the CNM effects: it can be reproduced assuming Cronin effect for charm quarks [9]. A direct measurement of elliptic flow $v_{2}$ of $D^{0}$ will answer the question of heavy quark collectivity in $p+A$ collisions. High luminosity $p+A$ collisions at AFTER will allow quantifying the CNM effects and measuring $v_{2}$ with high precision to address these two issues.

In this paper we present estimates of the precision expected for open heavy flavor measurements at the proposed fixed target experiment at LHC (AFTER@LHC). We first describe the simulation setup we use and then present and discuss estimates for physical observables for open charm and open bottom in $p+A$ collisions at $\sqrt{s_{N N}}=115 \mathrm{GeV}$ and $\mathrm{Pb}+\mathrm{Pb}$ collisions at $\sqrt{s_{N N}}=72 \mathrm{GeV}$. We argue that these measurements at AFTER will address open questions about heavy quark collectivity in $p+A$ collisions and energy dependence of the heavy quark interactions with a nuclear matter.

\section{Centrality Estimation}

We estimate centrality bins in $p+\mathrm{Pb}$ collisions at $\sqrt{s_{N N}}=$ $115 \mathrm{GeV}$ and $\mathrm{Pb}+\mathrm{Pb}$ at $\sqrt{s_{N N}}=72 \mathrm{GeV}$ using Glauber Monte Carlo calculations with the PHOBOS Glauber Monte Carlo $[10,11]$ version 2.1. We assume that the nucleon-nucleon cross section $\sigma_{N N}=37 \mathrm{mb}$ at $\sqrt{s_{N N}}=72 \mathrm{GeV}$ and the nucleonnucleon cross section $\sigma_{N N}=39 \mathrm{mb}$ at $\sqrt{s_{N N}}=115 \mathrm{GeV}$, and the rest of parameters have the default values.

Centrality bins are defined as percentiles of hadronic cross sections. Figure 1 shows impact parameter distributions in $p$-Pb collisions at $\sqrt{s_{N N}}=115 \mathrm{GeV}$ and (b) $\mathrm{Pb}$ - $\mathrm{Pb}$ collisions at $\sqrt{s_{N N}}=72 \mathrm{GeV}$. Tables 1 and 2 show the average number of participants $\left\langle N_{\text {part }}\right\rangle$ and binary collisions $\left\langle N_{\text {bin }}\right\rangle$ and their root mean square (RMS) values for those two systems for the centrality classes.

\section{Simulation Setup}

3.1. Detector Setup. We consider a detector with similar apparatus as the $\mathrm{LHCb}$ experiment [12]. We assume rapidity coverage in the laboratory frame of $2<y_{\mathrm{Lab}}<5$ and similar particle detector capabilities and efficiencies (muon detection capability and precise microvertexing detector). The efficiencies reported by LHCb $[13,14]$ are used in our estimates (Figure 2). We consider two experimental techniques for open heavy flavor measurements: $D^{0}$ measurements via secondary vertex reconstruction and $B \rightarrow J / \psi$ determination via a pseudo-lifetime method.

Since both $D^{0}$ and $J / \psi$ from B-hadron decays are measured at the forward rapidity in a laboratory frame with a precise vertex detector, a large boost will strongly suppress the background. Thus we neglect the background contribution to the statistical uncertainties in our estimations. The large boost also allows for $D$ and $B$ mesons and $B \rightarrow J / \psi$ measurements down to zero $p_{T}$ via displaced vertex reconstruction. 
TABLE 2: Average number of participants $\left\langle N_{\text {part }}\right\rangle$ and binary collisions $\left\langle N_{\text {bin }}\right\rangle$ in $\mathrm{Pb}-\mathrm{Pb}$ collisions at $\sqrt{\boldsymbol{s}_{N N}}=72 \mathrm{GeV}$ for centrality classes defined as percentiles of the hadronic cross section. The mean values and the RMS are obtained with a Glauber Monte Carlo calculation.

\begin{tabular}{|c|c|c|c|c|c|c|}
\hline Centrality & $b_{\min }[\mathrm{fm}]$ & $b_{\max }[\mathrm{fm}]$ & $\left\langle N_{\text {bin }}\right\rangle$ & $\operatorname{RMS}\left(N_{\text {bin }}\right)$ & $\left\langle N_{\text {part }}\right\rangle$ & $\operatorname{RMS}\left(N_{\text {part }}\right)$ \\
\hline $0-10 \%$ & 0 & 4.80 & 884.0 & 128.0 & 338.5 & 34.0 \\
\hline $10-20 \%$ & 4.80 & 6.77 & 557.2 & 82.2 & 241.8 & 25.6 \\
\hline $20-30 \%$ & 6.77 & 8.30 & 346.5 & 58.9 & 170.8 & 20.8 \\
\hline $30-40 \%$ & 8.30 & 9.59 & 206.2 & 42.9 & 117.1 & 17.2 \\
\hline $40-50 \%$ & 9.59 & 10.72 & 115.4 & 30.6 & 76.7 & 14.6 \\
\hline $50-60 \%$ & 10.72 & 11.75 & 59.4 & 20.6 & 47.0 & 12.0 \\
\hline $60-70 \%$ & 11.75 & 12.69 & 28.0 & 13.0 & 26.6 & 9.5 \\
\hline $70-80 \%$ & 12.69 & 13.58 & 12.2 & 7.5 & 13.7 & 6.8 \\
\hline $80-90 \%$ & 13.58 & 14.55 & 5.2 & 4.0 & 6.8 & 4.2 \\
\hline $90-100 \%$ & 14.55 & 20.00 & 2.3 & 1.8 & 3.5 & 2.1 \\
\hline
\end{tabular}

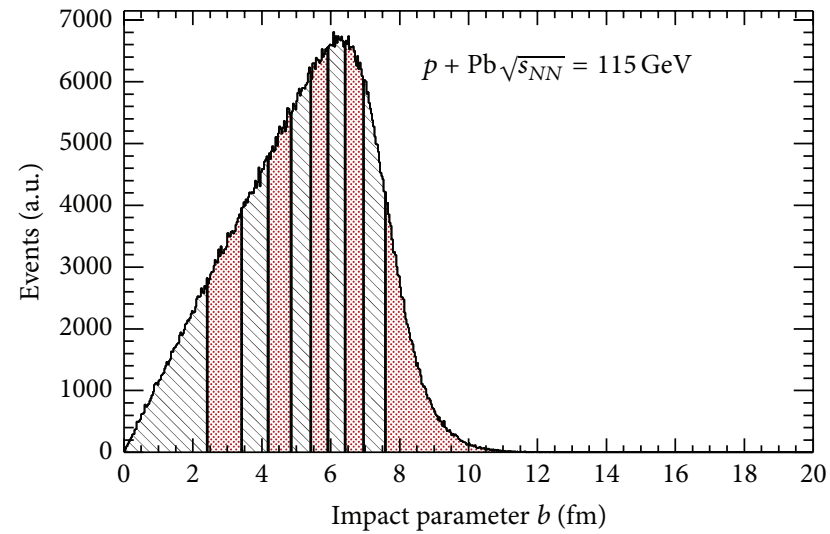

\begin{tabular}{|c|c|c|}
\hline Q 0-10\% & $\nabla 40-50 \%$ & 70-80\% \\
\hline 闵 10-20\% & 阅 50-60\% & Q 80-90\% \\
\hline$\triangle 20-30 \%$ & $\nabla 60-70 \%$ & 邋 90-100\% \\
\hline
\end{tabular}

(a)

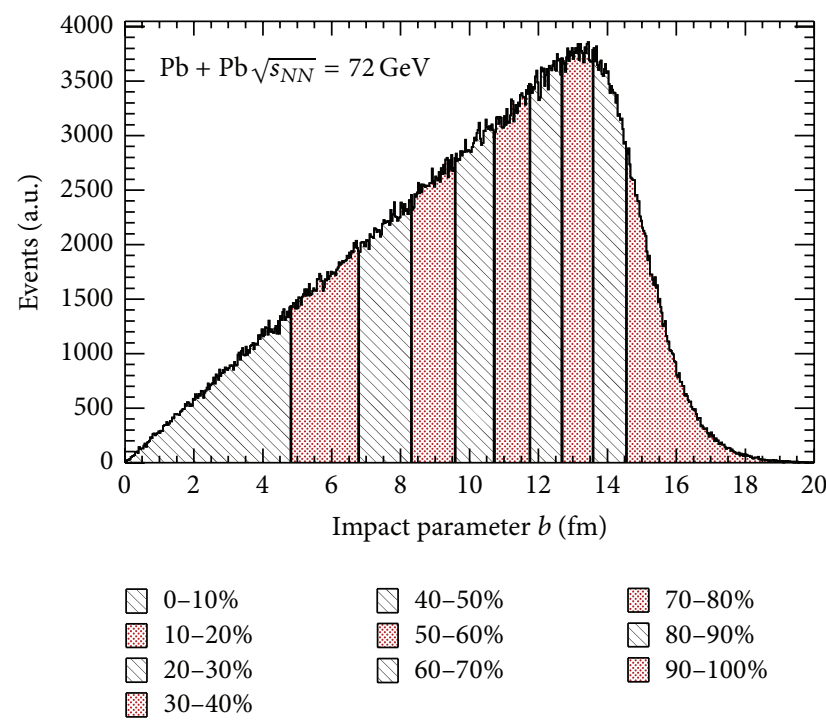

(b)

FIGURE 1: Impact parameter distribution for percentiles of the hadronic cross section obtained from a Glauber Monte Carlo calculation in (a) $p-\mathrm{Pb}$ collisions at $\sqrt{s_{N N}}=115 \mathrm{GeV}$ and (b) Pb-Pb collisions at $\sqrt{s_{N N}}=72 \mathrm{GeV}$.

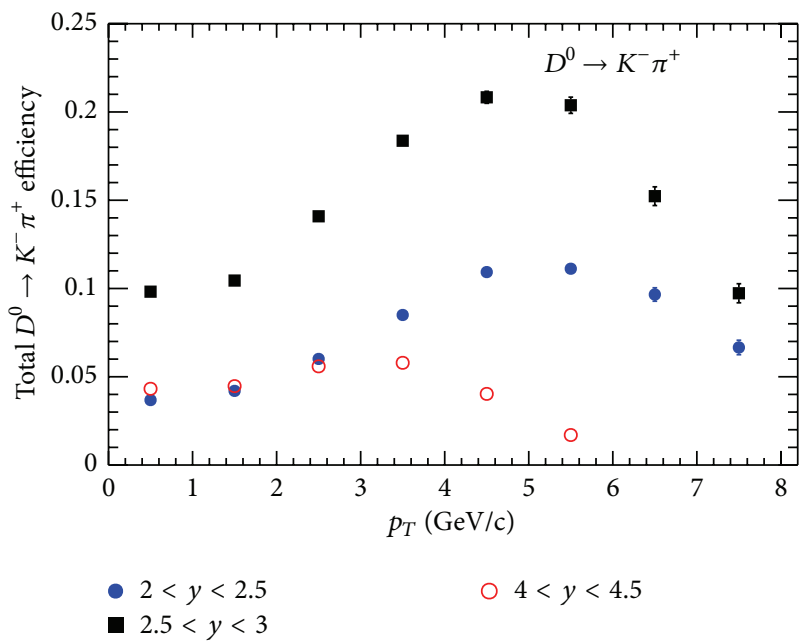

(a)

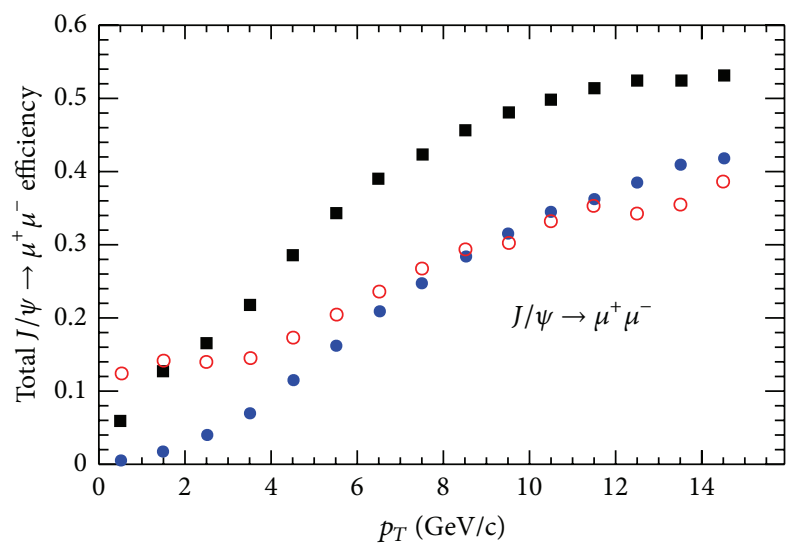

- $2<y<2.5$

- $2.5<y<3$
○ $4<y<4.5$

(b)

FIGURE 2: Total reconstruction efficiency for (a) $D^{0}[13]$ and (b) $J / \psi[14]$ in the LHCb experiment. 


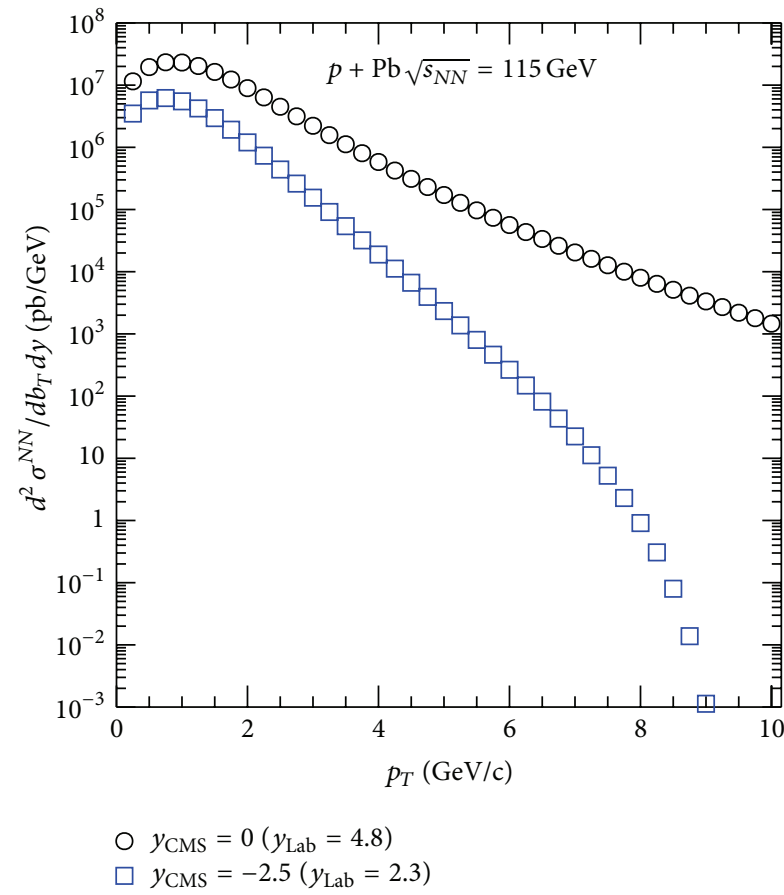

(a)

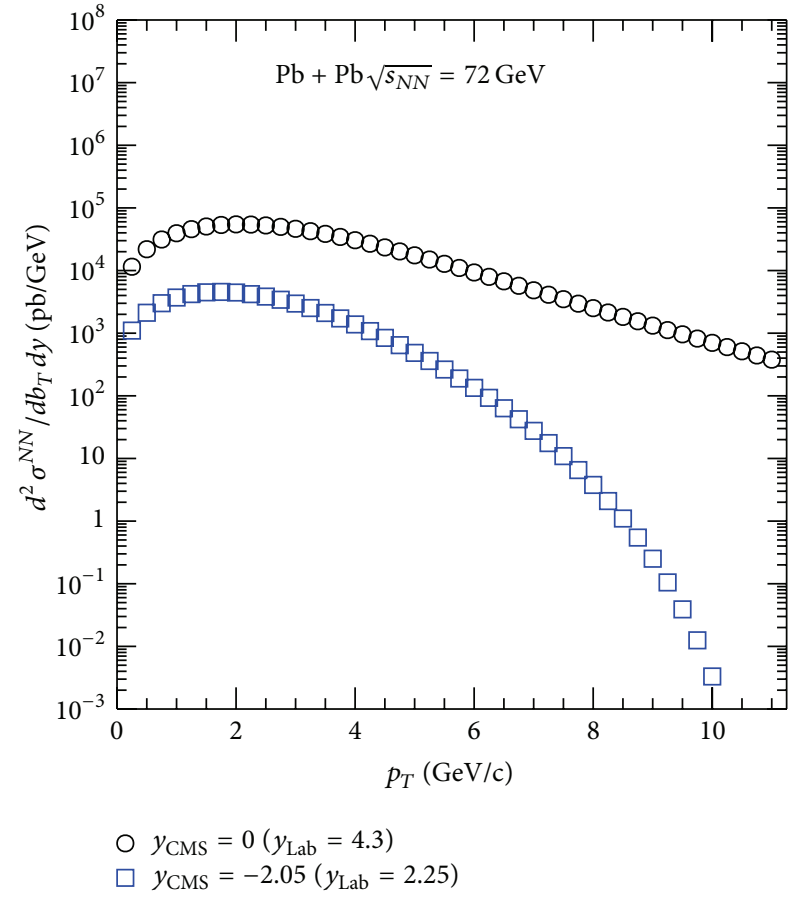

(b)

FIGURE 3: Charm quark differential cross section per nucleon-nucleon collision as a function of $p_{T}$ in $p+\mathrm{Pb}$ and $\mathrm{Pb}+\mathrm{Pb}$ collisions.

3.2. Heavy Quarks Production Cross Sections. We obtain charm and bottom quark production cross sections using FONLL (fixed-order plus next-to-leading logs) program [15, 16], version 1.3.3 [17]. We run the calculation assuming charm quark mass $m_{c}=1.5 \mathrm{GeV} / \mathrm{c}^{2}$ and bottom mass $m_{b}=$ $4.75 \mathrm{GeV} / \mathrm{c}^{2}$ using CTEQ6.6 [18] parton distribution function convoluted with EPS09 [19] shadowing parametrization (central value). Other parameters (scales) have the default values.

Figures 3 and 4 show charm and bottom quarks differential cross section per nucleon-nucleon collision as a function of $p_{T}$ in $p+\mathrm{Pb}$ and $\mathrm{Pb}+\mathrm{Pb}$ collisions. Only central values from FONLL are shown. Since experimental data for heavy flavor production at RHIC energies are consistent with upper limit on the FONLL calculations $[20,21]$ we believe that the results in Figures 3 and 4 provide us with conservative estimates of heavy quark cross section for those energies.

3.3. Charmed and Bottom Mesons Production. In the experiments we measure the final-state particles $\left(D^{0}\right.$, B-meson or $J / \psi$ from B-meson decays). In this study we made a few simplifications to estimate the expected yields based on charm and bottom cross sections. For $D^{0}$ meson, we assume that it has approximately the same $p_{T}$ spectrum as charm quarks. Charm hadronization ratio to $D^{0}$ is 0.565 [22] and $D^{0}$ will be measured via $D^{0} \rightarrow K^{-} \pi^{+}$(branching ratio $\mathrm{BR}=3.8 \%)$ via secondary vertex reconstruction. We used TPythia8Decayer from the ROOT framework [23] to simulate $B \rightarrow J / \psi$ decays. For the input, we assumed that $B^{ \pm}, B_{S}$, and $B^{0}$ have the same $p_{T}$ spectrum as bottom quarks, rapidity has a uniform distribution in narrow bins we have used $(\Delta y=0.5)$, and the hadronization fraction to $\mathrm{B}$ meson is $f(b \rightarrow B)=0.764\left(f\left(b \rightarrow B^{0}\right)=f\left(b \rightarrow B^{ \pm}\right)=\right.$ $(33.7 \pm 2.2) \%$ and $\left.f\left(b \rightarrow B_{S}\right)=(9.0 \pm 0.9) \%[24]\right)$. The branching ratio for $B \rightarrow J / \psi+X$ is taken as $\mathrm{BR}=1.16 \%$. Figure 4(b) shows an input bottom quark spectrum and $J / \psi$ from bottom meson decays for $|y|<0.25$. At the energy of $\sqrt{s_{N N}}=115 \mathrm{GeV}$, we expect bottom quark production with $p_{T}$ up to $11 \mathrm{GeV} / \mathrm{c}$, which allows for $B \rightarrow J / \psi$ measurement with $p_{T}$ up to $10 \mathrm{GeV} / \mathrm{c}$.

To calculate the production rates, we take an integrated luminosity $\mathscr{L}=160 \mathrm{pb}^{-1}$ for $p+\mathrm{Pb}$ collisions at $\sqrt{s_{N N}}=$ $115 \mathrm{GeV}$ and $\mathscr{L}=7 \mathrm{nb}^{-1}$ for $\mathrm{Pb}+\mathrm{Pb}$ collisions at $\sqrt{s_{N N}}$ $=72 \mathrm{GeV}$ [25]. Finally, we apply the charmed meson and $J / \psi$ reconstruction efficiencies reported by $\mathrm{LHCb}$ (Figure 2) to the simulated $D^{0}$ and $B \rightarrow J / \psi$ distributions to estimate the yields and statistical uncertainties expected in the experiment.

3.4. Energy Loss of Charm Quarks. The suppression of open heavy flavor production observed at RHIC is comparable to that for light hadrons. This was a surprising result at the beginning because models which described well the light flavor data assuming gluon radiation (radiative energy loss, $\left.d E / d x_{\text {rad }}\right)$ predicted that the heavy quark production will be less suppressed than light partons [26]. This led to introduction of collisional energy loss $d E / d x_{\text {coll }}$ due to binary interactions of partons with other objects in the QGP. One difficulty in modeling heavy quark energy loss is that the relative contributions of $d E / d x_{\text {coll }}$ and $d E / d x_{\text {rad }}$ are unknown. To understand better the interplay of these two processes, precise measurements of suppression of charm and bottom separately are necessary. We argue that measurements 


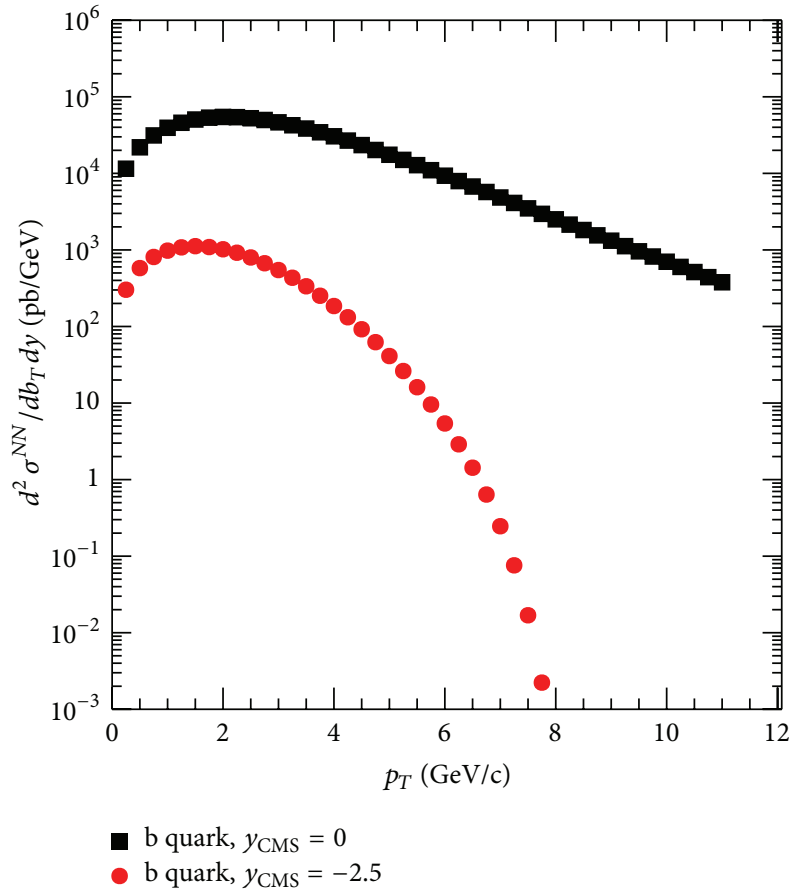

(a)

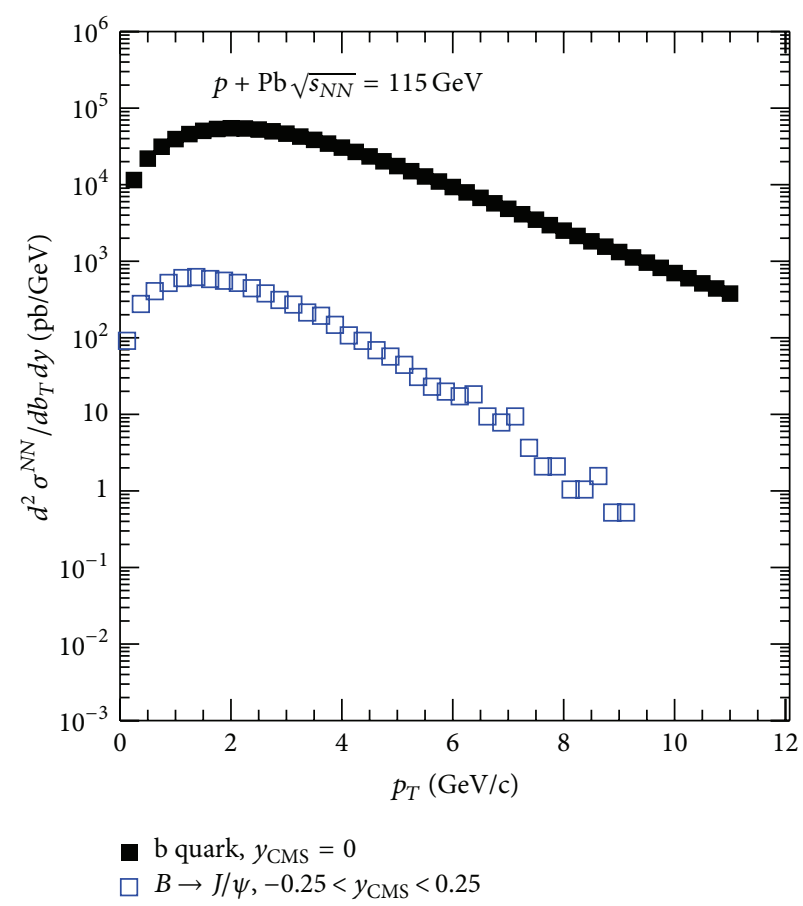

(b)

FIgURE 4: Bottom quark differential cross section per nucleon-nucleon collision as a function of $p_{T}$ in $p+\mathrm{Pb}$ collisions.

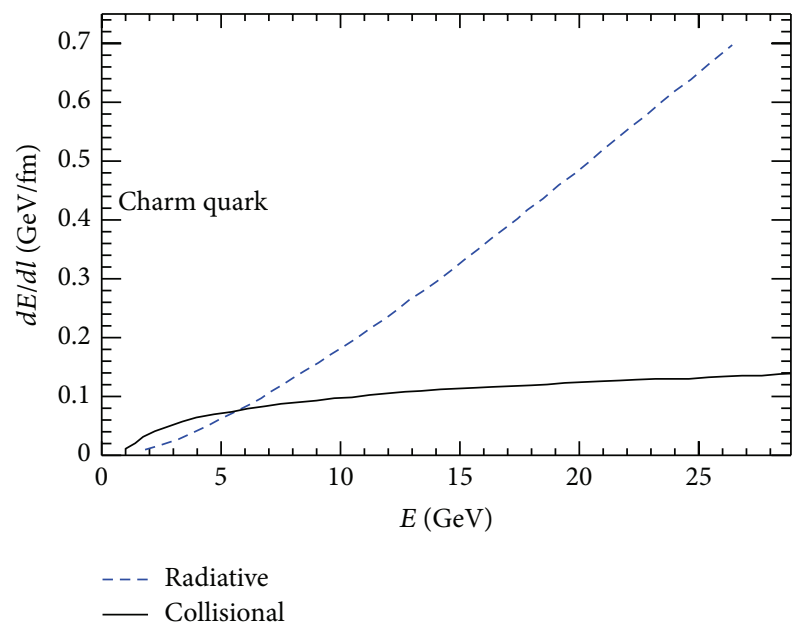

FIGURE 5: Radiative and collisional energy loss for charm quarks from [27] (model I) as a function of charm quark energy. Adopted from [27].

in $\mathrm{Pb}+\mathrm{Pb}$ collisions at $\sqrt{s_{N N}}=72 \mathrm{GeV}$ in different rapidity ranges can help to estimate what are the shares of $d E / d x_{\text {rad }}$ and $d E / d x_{\text {coll }}$ in the observed suppression.

To model charm quark energy loss, we use predictions from [27] for radiative and collisional energy loss of heavy quark. Figure 5 shows the expected energy loss per unit of distance traveled in a nuclear matter for charm quarks from model I in [27] with a coupling constant $\alpha_{s}=0.3$, an infrared regulator $\mu=0.4 \mathrm{GeV}$, and temperature $T=200 \mathrm{MeV}$ (see
[27] for details). For low energies of heavy quarks, $d E / d x_{\text {coll }}$ dominates but the radiative energy loss increases fast with increasing heavy quark energy and $d E / d x_{\text {rad }}$ overshadows $d E / d x_{\text {coll }}$ for $E>5 \mathrm{GeV}$.

A large acceptance of planned AFTER experiment allows probing different regimes of the energy loss, despite limited $D^{0} p_{T}$ range available experimentally, because charm quark at forward/backward rapidity will have a larger overall energy for the same $p_{T}$ compared to midrapidity.

To model the suppression of heavy quark production, we assume that the average path length in the nuclear matter $\langle L\rangle$ is approximately equal to the averaged transverse path length $\left\langle L_{x y}\right\rangle$ (distance traveled in a plane perpendicular to the beam axis) traversed by a quark from a production point to the edge of the created fireball. We adopted a definition of $\langle L\rangle=$ $\sqrt{\sigma_{x}^{2}+\sigma_{y}^{2}}$ from [28], where $\sigma_{x}^{2}$ and $\sigma_{y}^{2}$ are variances of $x$ and $y$ positions of the participant nucleons, respectively. $\sigma_{x}^{2}$ and $\sigma_{y}^{2}$ are obtained using PHOBOS Glauber Monte Carlo and $\langle L\rangle=4.104 \mathrm{fm}$ for $0-10 \%$ most central collisions. We use a nuclear modification factor $R_{A A}$ to quantify the modification of the charm quark $p_{T}$ spectrum due to energy loss:

$$
R_{A A}\left(p_{T}\right)=\frac{N_{E_{\text {Loss }}^{A A}}^{A A}\left(p_{T}\right)}{N_{\text {un.mod }}^{A A}\left(p_{T}\right)},
$$

where $N_{E_{\text {Loss }}}$ is a yield with energy loss applied and $N_{\text {un.mod }}$ is a heavy quark yield without any energy loss for a given centrality class and a given rapidity bin.

Figure 6 shows a nuclear modification factor $R_{A A}$ (central value) at midrapidity (Figure 6(a)) and backward rapidity 


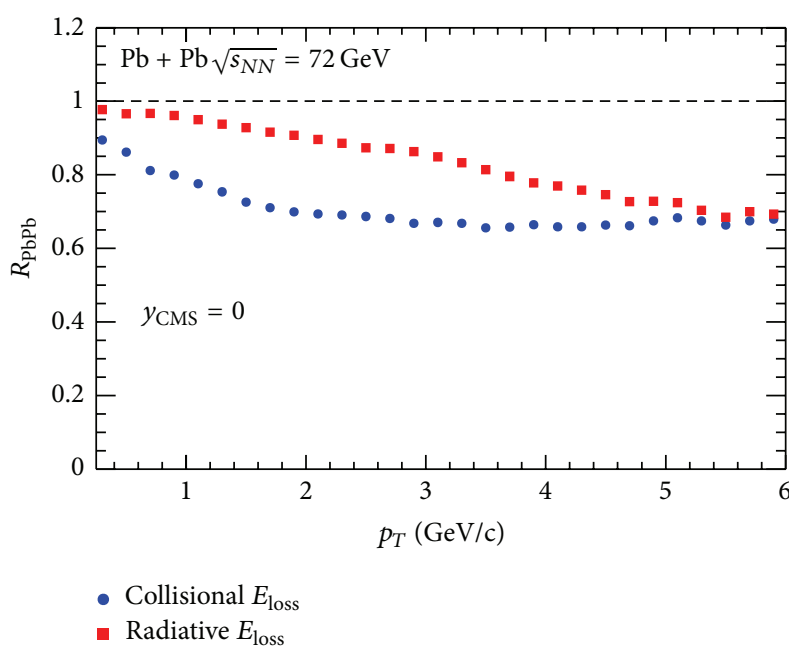

(a)

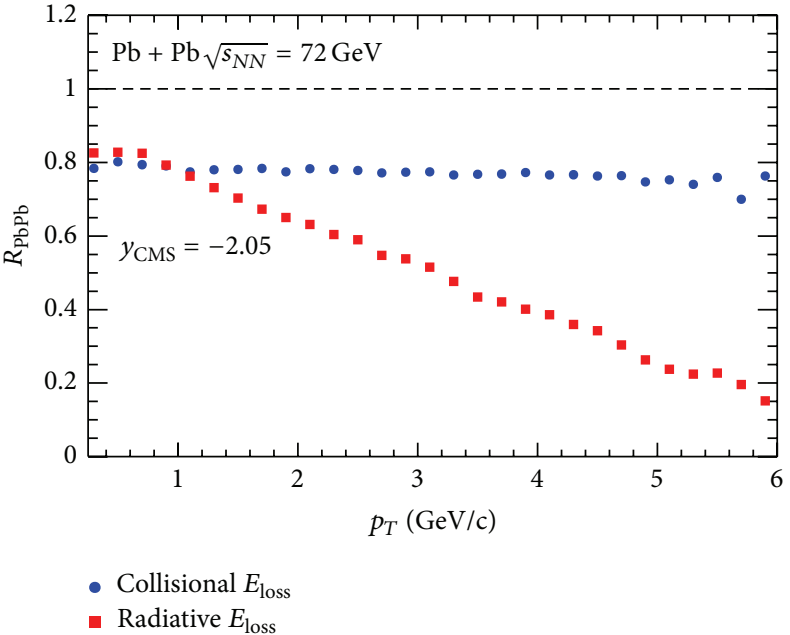

(b)

FiguRE 6: Nuclear modification factor $R_{A A}$ (central value) for charm quark in $\mathrm{Pb}+\mathrm{Pb}$ collisions at $\sqrt{\boldsymbol{s}_{N N}}=72 \mathrm{GeV}$ at midrapidity $\left(y_{\mathrm{CMS}}=0\right)$ and forward rapidity $\left(y_{\mathrm{CMS}}=-2.05\right)$ for a radiative and collisional energy loss from Figure 5 .

(Figure 6(b)) for $0-10 \%$ most central $\mathrm{Pb}+\mathrm{Pb}$ collisions expected for $d E / d x_{\text {coll }}$ and $d E / d x_{\text {rad }}$ shown in Figure 5. The difference between $d E / d x_{\text {rad }}$ and $d E / d x_{\text {coll }}$ is more pronounced at backward rapidity, even at relatively low $p_{T}$, because quarks have much larger energy due to larger longitudinal momentum. Measurement at these two rapidity ranges simultaneously could help to pin down interplay of these two mechanisms.

\section{Nuclear Modification Factors and Elliptic Flow in $\boldsymbol{p}+\mathbf{P b}$ Collisions at $\sqrt{\left(s_{N N}\right)}=115 \mathrm{GeV}$}

The sampled luminosity calculation in $p+p$ collisions is a significant source of the uncertainty when an energy loss is studied comparing heavy ion and $p+p$ collisions. Alternatively, we can use a central-to-peripheral ratio $R_{\mathrm{CP}}$ to quantify modification of the yields in $p+A$ or $A+A$ collisions:

$$
R_{\mathrm{CP}}\left(p_{T}\right)=\frac{N_{\text {bin }}^{\text {Perip }}}{N_{\text {bin }}^{\text {Central }}} \frac{N^{\text {Central }}\left(p_{T}\right)}{N^{\text {Perip }}\left(p_{T}\right)},
$$

where $N^{\text {Central }}$ and $N^{\text {Perip }}$ are yields in central and peripheral $A+A$ collisions, respectively, for a given rapidity range and $N_{\text {bin }}^{\text {Central }}$ and $N_{\text {bin }}^{\text {Perip }}$ are the average numbers of binary collisions for central and peripheral collisions. We use $0-10 \%$ most central collisions as a central bin and $60-80 \%$ bin as a baseline in $R_{\mathrm{CP}}$ calculations.

Figure 8(a) shows the expected statistical uncertainties for nuclear modification factor $R_{\mathrm{CP}}$ for $D^{0}$ at midrapidity and backward rapidity. Figure 7 presents the results for $B \rightarrow J / \psi$. The expected precision will allow a precision study of the cold nuclear matter effects for both charm and bottom for $p_{T}<6 \mathrm{GeV} / \mathrm{c}$. Figure 8(b) shows statistical uncertainties for elliptic flow measurement. High luminosity data expected from AFTER would give a precise answer if there are any signs

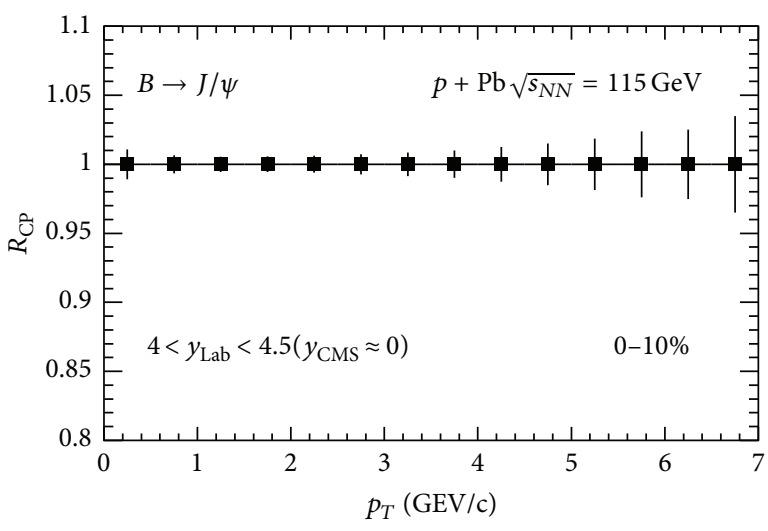

Figure 7: Expected statistical uncertainty of $R_{\mathrm{CP}}$ for $B \rightarrow J / \psi$ in $p$ $+\mathrm{Pb}$ collisions at $\sqrt{s_{N N}}=115 \mathrm{GeV}$.

of collective behavior of charm quarks in $p+A$ collisions at the intermediate energy at RHIC.

\section{Nuclear Modification Factor for Charmed Meson in $\mathbf{P b}+\mathbf{P b}$ Collisions at $\sqrt{s_{N N}}=72 \mathrm{GeV}$}

Figure 9 shows the expected precision of $R_{\mathrm{CP}}$ for charmed meson as a function of $p_{T}$ at midrapidity and backward rapidity. We show estimates for pure collisional and radiative energy loss scenarios based on Figure 5. The expected integrated luminosity for a single year will allow addressing the question if charm production is suppressed at high transverse momentum at $\sqrt{s_{N N}}=72 \mathrm{GeV}$. The comparison of results at different rapidity ranges will help to pin down the interplay of energy loss mechanisms at this energy range. The measurements of $D^{0}$ suppression for various collisions systems (which is feasible in a multiple-year physics program at AFTER) are even more interesting since they will allow 


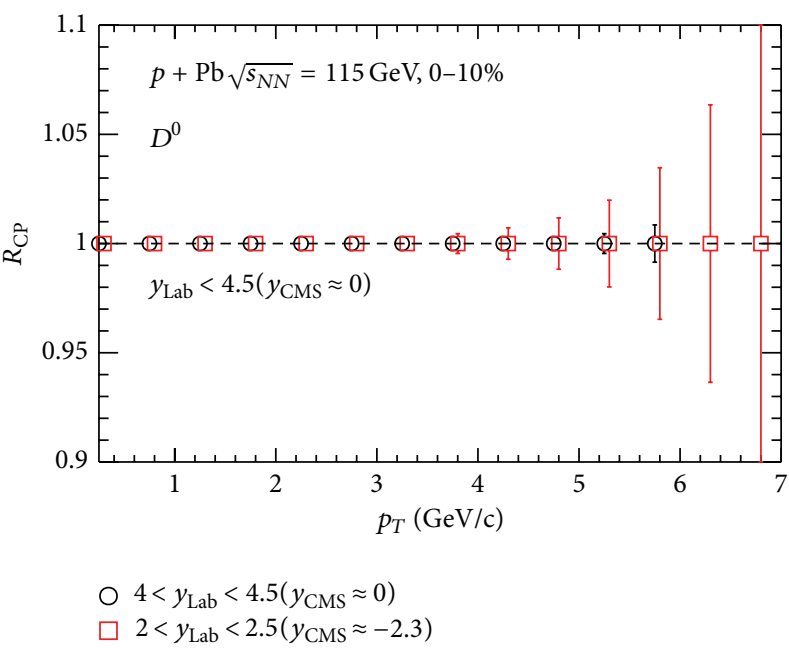

(a)

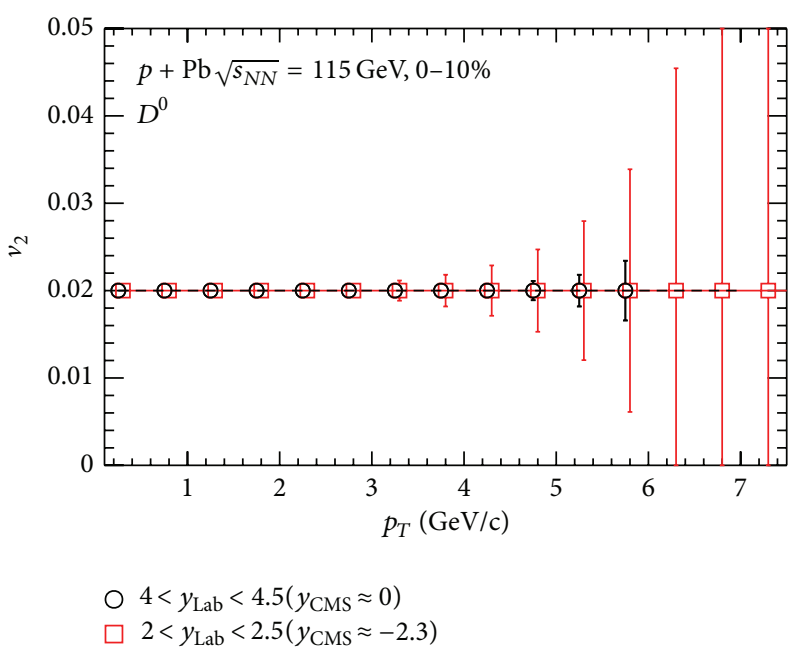

(b)

FIGURE 8: Expected statistical uncertainties of (a) $D^{0} R_{\mathrm{CP}}$ and (b) $D^{0}$ elliptic flow in $p+\mathrm{Pb}$ collisions at $\sqrt{s_{N N}}=115 \mathrm{GeV}$.

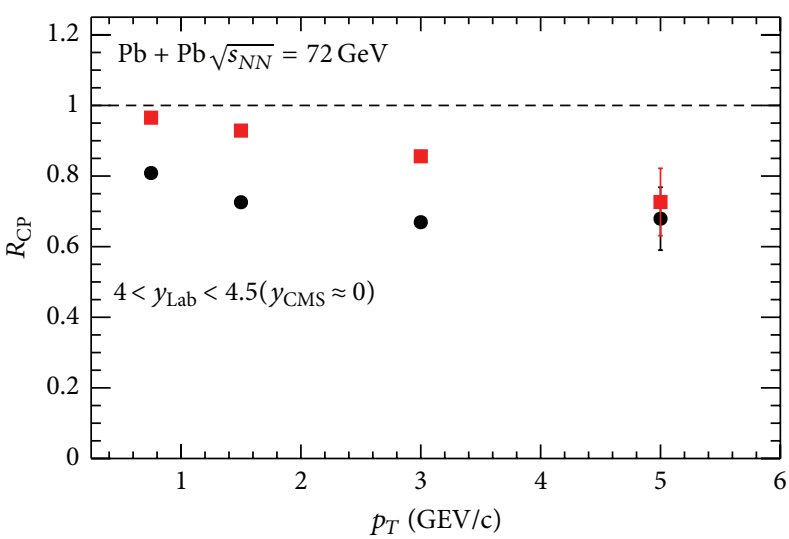

- Collisional $E_{\text {loss }}$

- Radiative $E_{\text {loss }}$

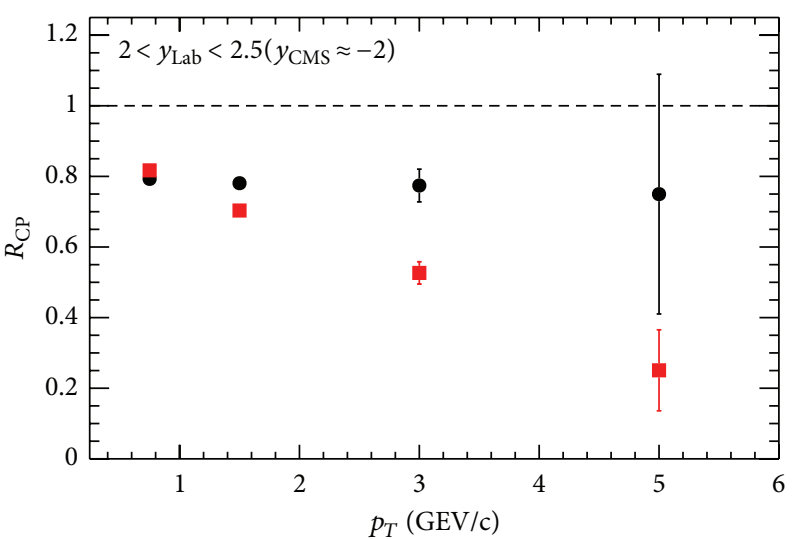

- Collisional $E_{\text {loss }}$

- Radiative $E_{\text {loss }}$

(a)

(b)

FIGURE 9: Expected statistical uncertainties of nuclear modification factor $R_{\mathrm{CP}}$ for $D^{0}$ meson in $\mathrm{Pb}+\mathrm{Pb}$ collisions at $\sqrt{s_{N N}}=72 \mathrm{GeV}$.

studying the energy loss as a function of path length and energy density of the created system.

\section{Summary}

We presented prospects for open charm and bottom production measurements in a fixed target experiment at LHC. We argue that such measurements will address important open issues about interactions of heavy quarks with the nuclear matter. High luminosity $p+A$ and $\mathrm{Pb}+\mathrm{Pb}$ data will help answer questions if there is a collective behavior of heavy quark in $p+A$ collisions at RHIC and what is the mechanism of energy loss of heavy quarks. Measurements at $\sqrt{s_{N N}}=$ $72 \mathrm{GeV}$ can shed new light on energy dependence of heavy quark interaction with the nuclear matter and thus on the phase diagram of nuclear matter.

\section{Conflict of Interests}

The author declares that there is no conflict of interests regarding the publication of this paper.

\section{Acknowledgment}

This work was supported in part by the Foundation for Polish Science Grant HOMING PLUS/2013-7/8.

\section{References}

[1] R. Rapp and H. van Hees, "Heavy quarks in the Quark-Gluon plasma," in Quark-Gluon Plasma, vol. 4, chapter 3, p. 111, World Scientific Publishers, 2010, http://arxiv.org/abs/0903.1096. 
[2] S. Voloshin and Y. Zhang, "Flow study in relativistic nuclear collisions by Fourier expansion of azimuthal particle distributions," Zeitschrift für Physik C, vol. 70, no. 4, pp. 665-671, 1996.

[3] L. Adamczyk, J. K. Adkins, G. Agakishiev et al., "Elliptic flow of non-photonic electrons in Au+Au collisions at $\sqrt{s_{N N}}=200$, 62.4 and $39 \mathrm{GeV}$," http://arxiv.org/abs/1405.6348.

[4] A. Adare, C. Aidala, N. N. Ajitanand et al., "Quadrupole anisotropy in dihadron azimuthal correlations in central $\mathrm{d}+\mathrm{Au}$ Collisions at $\sqrt{s_{N N}}=200 \mathrm{GeV}$," Physical Review Letters, vol. 111, Article ID 212301, 2013.

[5] S. Chatrchyan, V. Khachatryan, A. M. Sirunyan, and A. Tumasyan, "Multiplicity and transverse momentum dependence of two- and four-particle correlations in $\mathrm{pPb}$ and $\mathrm{PbPb}$ collisions," Physics Letters B, vol. 724, pp. 213-240, 2013.

[6] B. I. Abelev, J. Adam, R. A. Bertens et al., "Long-range angular correlations of $\pi, \mathrm{K}$ and $\mathrm{p}$ in $\mathrm{p}-\mathrm{Pb}$ collisions at $\sqrt{s_{N N}}=5.02$ TeV," Physics Letters B, vol. 726, no. 1-3, pp. 164-177, 2013.

[7] A. Adare, S. Afanasiev, C. Aidalam et al., "Cold-nuclear-matter effects on heavy-quark production in $d+$ Au collisions at $\sqrt{s_{N N}}=200$ GeV," Physical Review Letters, vol. 109, Article ID 242301, 2012.

[8] A. M. Sickles, "Possible evidence for radial flow of heavy mesons in d + Au collisions," Physics Letters B, vol. 731, pp. 51-56, 2014.

[9] D. Kikola and A. Lipiec, "Estimate of cold nuclear matter effects on bottom production in $d$ + Au collisions at $\sqrt{s_{N N}}=200 \mathrm{GeV}$," http://arxiv.org/abs/1410.6503.

[10] B. Alver, M. Baker, C. Loizides, and P. Steinberg, “The PHOBOS Glauber Monte Carlo," http://arxiv.org/abs/0805.4411.

[11] C. Loizides, J. Nagle, and P. Steinberg, "Improved version of the PHOBOS Glauber Monte Carlo," http://arxiv.org/abs/ 1408.2549 .

[12] A. A. Alves Jr., L. M. A. Filho, A. F. Barbosa et al., "The LHCb detector at the LHC," Journal of Instrumentation, vol. 3, Article ID S08005, 2008.

[13] A. V. Kozlinskiy, Outer tracker calibration and open charm production cross section measurement at LHCb [Ph.D. thesis], Vrije Universiteit, CERN-THESIS-2012-338, Amsterdam, The Netherlands, 2012.

[14] R. Aaij, B. Adeva, M. Adinolfi et al., "Measurement of $J / \psi$ production in $p p$ collisions at $\sqrt{s}=7 \mathrm{TeV}$," The European Physical Journal C, vol. 71, article 1645, 2011.

[15] M. Cacciari, M. Greco, and P. Nason, "The $p_{T}$ spectrum in heavy-flavour hadroproduction," Journal of High Energy Physics, vol. 1998, no. 5, article 007, 1998.

[16] M. Cacciari, S. Frixione, N. Houdeau, M. L. Mangano, P. Nason, and G. Ridolfi, "Theoretical predictions for charm and bottom production at the LHC," Journal of High Energy Physics, vol. 2012, no. 10, article 137, 2012.

[17] P. N. M. Cacciari and S. Frixione, FONLL, version 1.3.3, http://cacciari.web.cern.ch/cacciari/fonll.

[18] P. M. Nadolsky, H.-L. Lai, Q.-H. Cao et al., "Implications of CTEQ global analysis for collider observables," Physical Review D, vol. 78, Article ID 013004, 2008.

[19] K. Eskola, H. Paukkunena, and C. Salgado, "EPS09-global NLO analysis of nuclear PDFs and their uncertainties," Nuclear Physics A, vol. 830, no. 1-4, pp. 599c-602c, 2009.

[20] L. Adamczyk, G. Agakishiev, M. M. Aggarwal et al., "Measurements of $D^{0}$ and $D^{*}$ production in $p+p$ collisions at $\sqrt{s}=200$ GeV," Physical Review D, vol. 86, Article ID 072013, 2012.

[21] Z. Ye, "Open charm hadron production in $\mathrm{p}+\mathrm{p}, \mathrm{Au}+\mathrm{Au}$ and U+U collisions at STAR," Nuclear Physics A, vol. 931, pp. 520524, 2014.
[22] C. Amsler, M. Doser, M. Antonelli et al., "Review of particle physics," Physics Letters B, vol. 667, no. 1-5, pp. 1-6, 2008.

[23] I. Antcheva, "ROOT-a C++ framework for petabyte data storage, statistical analysis and visualization," Computer Physics Communications, vol. 180, no. 12, pp. 2499-2512, 2009, http:// root.cern.ch.

[24] R. Aaij, C. Abellan Beteta, B. Adeva et al., "Measurement of b hadron production fractions in $7 \mathrm{TeV} p p$ collisions," Physical Review D, vol. 85, Article ID 032008, 2012.

[25] S. J. Brodsky, F. Fleuret, C. Hadjidakis, and J. P. Lansberg, "Physics opportunities of a fixed-target experiment using LHC beams," Physics Reports, vol. 522, no. 4, pp. 239-255, 2013.

[26] M. Djordjevic, M. Gyulassy, R. Vogt, and S. Wicks, "Influence of bottom quark jet quenching on single electron tomography of $\mathrm{Au}+\mathrm{Au}$," Physics Letters, Section B: Nuclear, Elementary Particle and High-Energy Physics, vol. 632, no. 1, pp. 81-86, 2006.

[27] J. Aichelin, P. B. Gossiaux, and T. Gousset, "Collisional and radiative energy loss of heavy quarks," Journal of Physics: Conference Series, vol. 455, no. 1, Article ID 012046, 2013.

[28] H. Masui, B. Mohanty, and N. Xu, "Predictions of elliptic flow and nuclear modification factor from $200 \mathrm{GeV} \mathrm{U}+\mathrm{U}$ collisions at RHIC," Physics Letters B, vol. 679, no. 5, pp. 440-444, 2009. 

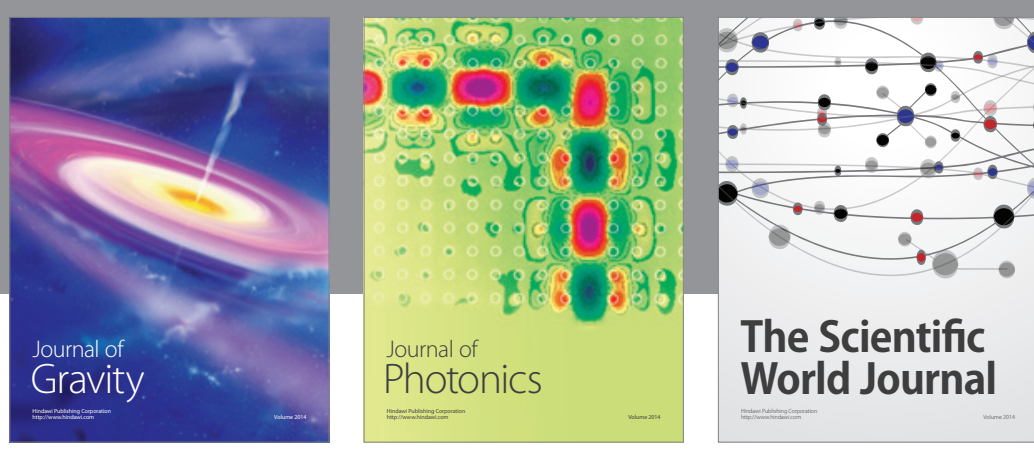

The Scientific World Journal
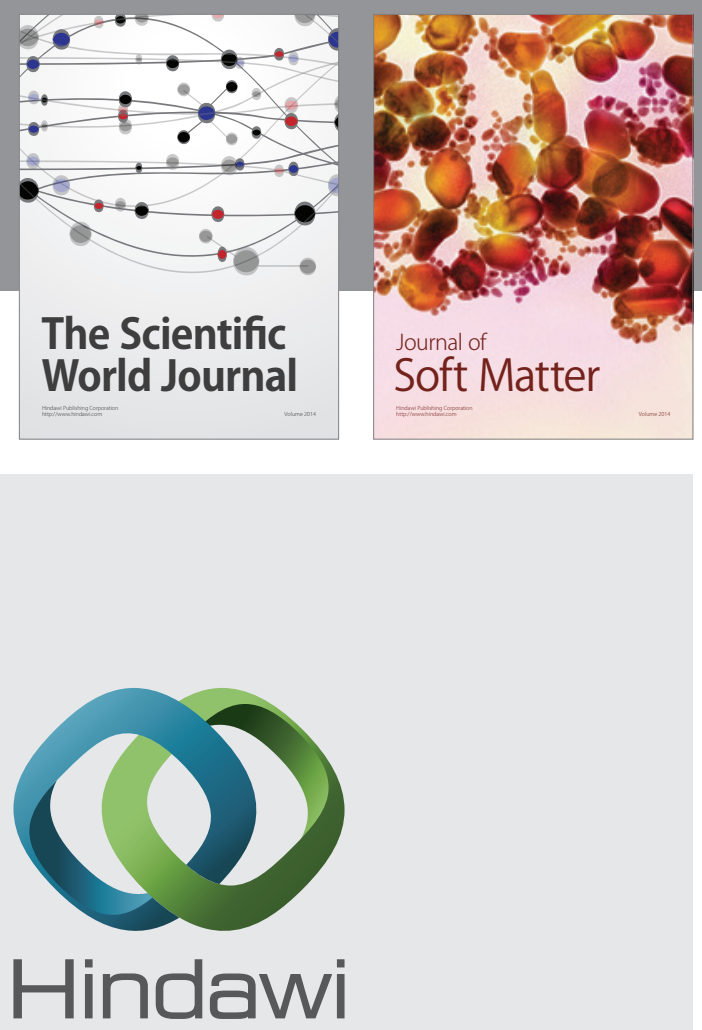

Submit your manuscripts at

http://www.hindawi.com

nternational Journal of

Statistical Mechanics
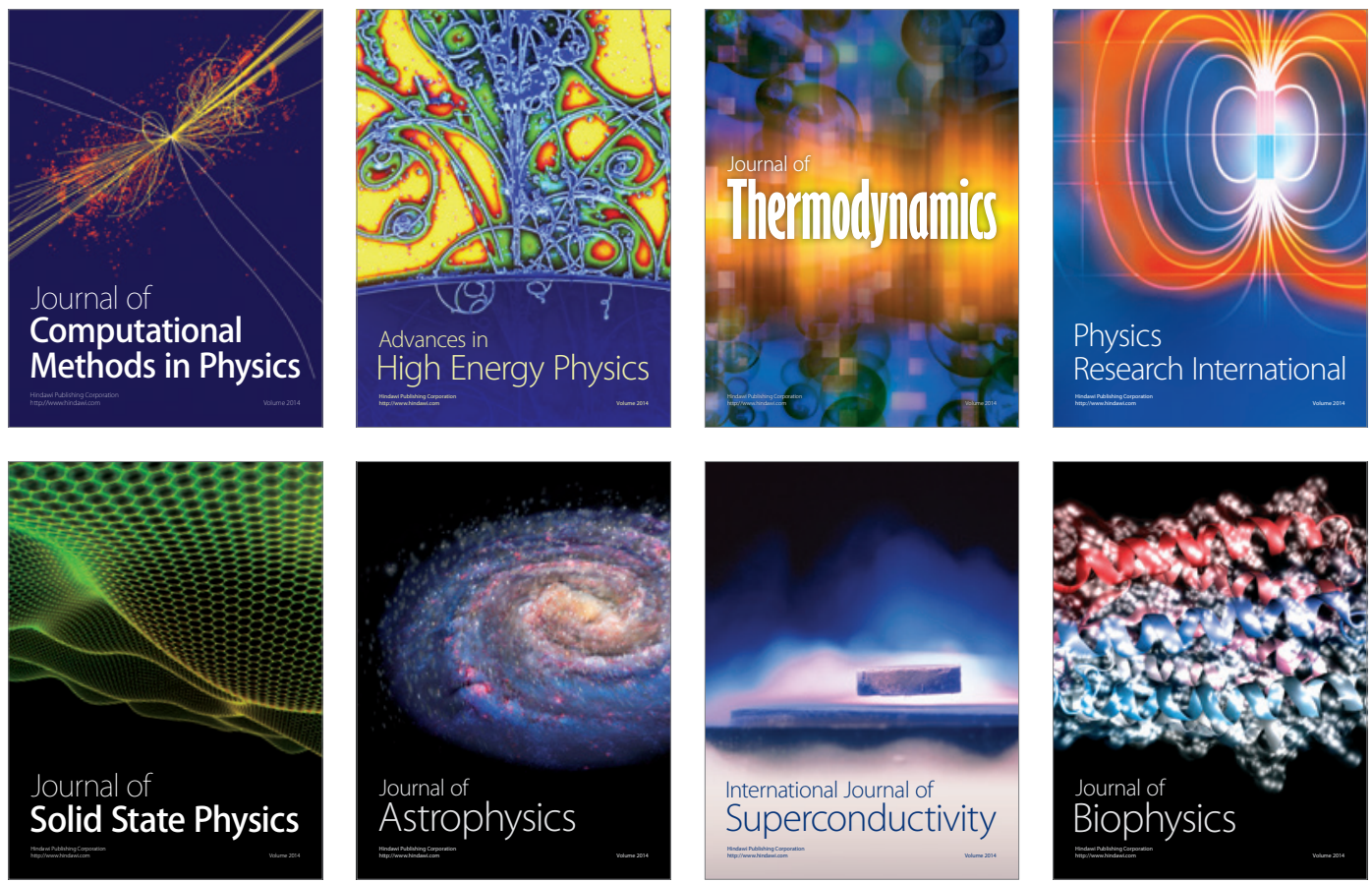
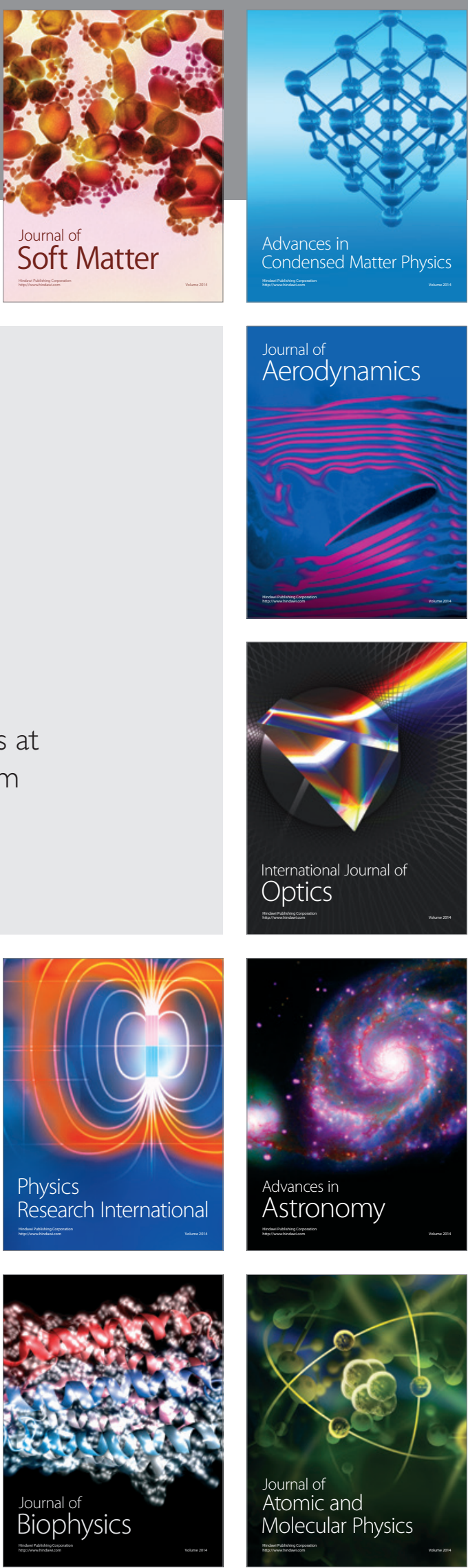